\title{
Possible Association between Middle Ear Cholesteatoma and A Single Nucleotide Polymorphism 538G >A in ABCC11, A Human Earwax Type Determinant
}

\author{
Kusunoki $\mathrm{T}^{1^{*}}$, Nakagawa $\mathrm{H}^{2}$, Tsukamoto $\mathrm{M}^{2}$, Toyoda $\mathrm{Y}^{3}$, Kamiya $\mathrm{K}^{4}$, Okada $\mathrm{H}^{4}$, Hara $\mathrm{S}^{4}$, Furukawa $\mathrm{M}^{4}$ and Ikeda $\mathrm{K}^{4}$ \\ ${ }^{1}$ Department of Otorhinolaryngology, Juntendo University of Medicine, Shizuoka Hospital, Shizuoka, Japan, \\ ${ }^{2}$ Department of Applied Biological Chemistry, Graduate School of Bioscience and Biotechnology, Chubu University, Japan \\ ${ }^{3}$ Department of Pharmacy, The University of Tokyo Hospital, Japan \\ ${ }^{4}$ Department of Otorhinolaryngology, Juntendo University of Medicine, Faculty of Medicine, Japan
}

"Corresponding author: Dr. Takeshi Kusunoki, Department of Otorhinolaryngology, Juntendo University of Medicine, Shizuoka Hospital 1129, Nagaoka Izunokuni-Shi, Shizuoka 410-2295, Japan, Tel: +81-55-948-3111, Fax: +81-55-948-5088; E-mail: ttkusunoki001@aol.com

Rec date: July 26, 2018; Acc date: September 05, 2018; Pub date: September 10, 2018

Copyright: (c) 2018 Kusunoki T, et al. This is an open-access article distributed under the terms of the creative commons attribution license, which permits unrestricted use, distribution, and reproduction in any medium, provided the original author and source are credited.

\begin{abstract}
Background and Objective: Humans have two types of earwax, classified as wet or dry, which are determined by a single nucleotide polymorphism (SNP) (rs17822931, 538G>A; Gly180Arg) in the ATP-binding cassette transporter $C 11$ (ABCC11) gene. The 538G/G and G/A, and the $A / A$ genotypes, are responsible for the wet and the dry type of earwax, respectively. The authors recently reported that the wet type of earwax was more often observed in patients with bilateral middle ear cholesteatoma than in those with unilateral middle ear cholesteatoma. Although the research population in the previous study was not large, the results, nevertheless, suggest a plausible association between the wet type of earwax and the risk for bilateral middle ear cholesteatoma. In this context, the authors hypothesized that $A B C C 11538 \mathrm{G}>\mathrm{A}$, a human earwax determinant, could also be related to the risk for bilateral middle ear cholesteatoma. In the present study, we examined the genotypes of $A B C C 11538 \mathrm{G}>\mathrm{A}$ in patients with cholesteatoma and compared genetic variations between cases with bilateral and unilateral cholesteatoma.
\end{abstract}

Methods: Blood samples from 31 patients with middle ear cholesteatoma were collected. After extraction of DNA from whole blood, the genomic region containing $A B C C 11538 \mathrm{G}>\mathrm{A}$ was specifically amplified using polymerase chain reaction (PCR). The genotypes at $A B C C 11538 \mathrm{G}>\mathrm{A}$ in each patient were then determined using a sequencing method. Experiments to determine the genotype of each patient in whom the $A B C C 11$ gene was amplified by PCR, and DNA sequencing, were performed in a masked manner.

Results: Earwax types in patients with cholesteatoma were in accordance with the genotypes of $A B C C 11$ $538 \mathrm{G}>\mathrm{A}$ without exceptions. In the bilateral cholesteatoma group, the number of patients with wet earwax tended to be greater than those in the unilateral cholesteatoma group.

Conclusion: Wet earwax might tend to cause bilateral rather than unilateral cholesteatoma in the Japanese population.

Keywords: $A B C C 11$ gene; Earwax; Ethnic difference; Middle ear cholesteatoma

\section{Introduction}

The risk for disease is generally affected by environmental and genetic factors. With regard to the development of middle ear cholesteatoma, a malignant disease of the ear, many researchers have focused on the middle ear environment. However, there have been few studies investigating the association between ear cholesteatoma and the environment in the external auditory canal. Moreover, intrinsic risk factors for middle ear cholesteatoma remain to be elucidated. Recently, based on an epidemiological association between the frequency of middle cholesteatoma and the wet type of earwax in humans, we hypothesized a possible link between the incidence of middle ear cholesteatoma and a single nucleotide polymorphism
(SNP) (rs17822931, 538G>A; Gly180Arg) in the human ABCC11 gene, a determinant of the human earwax type [1].

Genetic studies have demonstrated that the $A B C C 11$ 538G/G and G/A genotypes result in the wet type of earwax, while the 538A/A genotype is associated with the dry type of earwax [2,3]. Biochemical analyses revealed that the $538 \mathrm{~A}$ allele results in a non-functional $A B C C 11$ protein known as the $\operatorname{Arg} 180$ variant $[3,4]$. Interestingly, in Western populations, the allele frequency of $A B C C 11538 \mathrm{G}$ is higher than that in Asian populations [1,2]. Consistent with the geographical distribution of this earwax-determinant allele, the incidence of middle ear cholesteatoma is reportedly higher in Europeans than in East Asians, including Japanese [5-9]. Considering these previous findings, it is important to address whether $A B C C 11538 \mathrm{G}$ is related to the risk for cholesteatoma.

In this context, we investigated the genotypes of $A B C C 11538 \mathrm{G}>\mathrm{A}$ in patients with middle ear cholesteatoma. Our findings might 
Citation: Kusunoki T, Nakagawa H, Tsukamoto M, Toyoda Y, Kamiya K, et al. (2018) Possible Association between Middle Ear Cholesteatoma and A Single Nucleotide Polymorphism 538G>A in ABCC11, A Human Earwax Type Determinant. J Mol Genet Med 12: 362. doi: $10.4172 / 1747-0862.1000362$

Page 2 of 3

contribute to developing new diagnostic and therapeutic strategies for the prevention of cholesteatoma.

\section{Materials and Methods}

\section{Patients}

The present study was approved by the Juntendo University Committee for Ethics (Tokyo, Japan) concerned with the human genome. All cases involved acquired cholesteatoma. Under oral and written informed consent, blood samples were collected from 31 patients. Some of these subjects were studied in terms of the relationship between earwax type and cholesteatoma risk in our previous study [10], whereas, for the first time in the present study, all patients were examined for the genotypes of $A B C C 11538 \mathrm{G}>\mathrm{A}$.

\section{Genotyping}

All blood samples were collected in standard 2Na-EDTA-coated blood collection tubes. The samples were subjected to proteinase $\mathrm{K}$ digestion; genomic DNA was then isolated using phenol/chloroform extraction and, subsequently, by ethanol precipitation. Before sequencing, part of the $A B C C 11$ gene, including the $538 \mathrm{G}>\mathrm{A}$ allele, was amplified from the genomic DNA by polymerase chain reaction (PCR) with the following primer set: forward 5'aacaaagctcctggctagcaag- $3^{\prime}$, and reverse $5^{\prime}$-ccataaggtctacacctgagggtc- $3^{\prime}$. The amplicons were then subjected to genotyping of $A B C C 11538 \mathrm{G}>\mathrm{A}$ using a sequencing method reported in our previous study with minor modifications [3]. In brief, after ExoSAP-IT treatment of the amplifications, samples were prepared using a specific primer $\left(5^{\prime}-\right.$ tcctggctagcaagaactaggatg- $3^{\prime}$ or $5^{\prime}$-attccatggggaaaccaagtc- $3^{\prime}$ ) and BigDye Terminator3.1 (Applied Biosystems Inc., Foster City, CA, USA) according to the manufacturer's instructions. The sequence information obtained by the Autosequencer Model 3100 (Applied Biosystems Inc.) were aligned with an AutoAssembler (Applied Biosystems Inc.) and visualized using Sequencher 4.7 Demo (Hitachi Software Engineering Co., Ltd., Tokyo, Japan). Experiments to determine the genotype of each patient in whom the $A B C C 11$ gene was amplified by PCR, and DNA sequencing, were performed in a masked manner.

\section{Statistical tests}

Statistical analysis were performed using the chi-squared test; $\mathrm{p}<0.05$ was considered to be statistically significant in the present study.

\section{Results}

Among 31 patients who underwent $A B C C 11$ genotyping, dry earwax with the 538A/A and wet earwax with the 538G/A were found in $45.2 \%$ ( $14 / 31$ patients) and $54.8 \%$ (17/31 patients), respectively (Figure 1). Wet earwax with the $538 \mathrm{G} / \mathrm{G}$ was not found in the present study (Table 1). The incidence of bilateral middle ear cholesteatoma (including history of surgery for middle ear cholesteatoma) was subsequently assessed. The incidence of bilateral middle ear cholesteatoma was $41.4 \%$ ( $7 / 17$ patients) in the wet-type group and $21.4 \%$ (3/14 patients) in the dry-type group. Although incidence was higher in the former group, the difference was not statistically significant.

\section{Discussion}

Some researchers have suggested that, as an extrinsic factor, one cause of middle ear cholesteatoma could be a gas-exchange failure in the middle ear cavity $[11,12]$. However, there have been few reports addressing intrinsic factors in the origin of middle ear cholesteatoma. According to previous studies describing the allele frequencies of $A B C C 11538 \mathrm{G}>\mathrm{A}$ among different ethnic populations $[1,2,13]$, most Africans and Europeans (80-100\%) have the wet type of earwax.

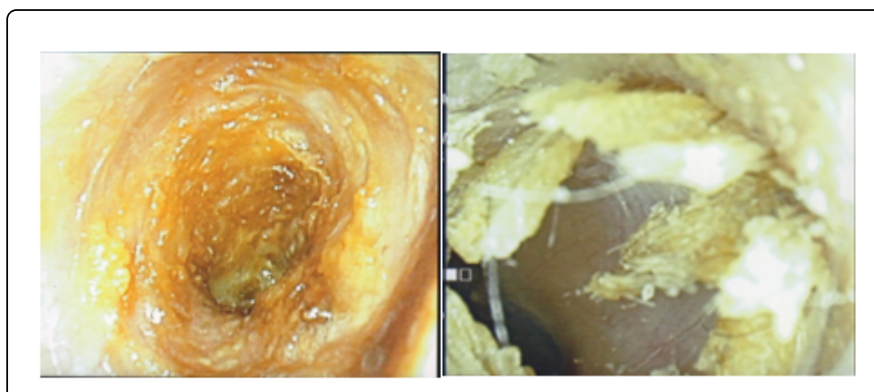

Figure 1: Representative images of earwax. Patients with the G/A genotypes in Table 1 exhibited the wet type of earwax (left), and patients with the A/A genotype exhibited the dry type (right).

\begin{tabular}{|l|l|l|l|}
\hline Ear Wax Type & \multicolumn{2}{|l|}{ Genotype of ABCC11 538>A } & Frequency \\
\hline Dry & A/A & $14 / 31$ & $45.2 \%$ \\
\hline Wet & G/A & $17 / 31$ & $54.8 \%$ \\
\hline $\begin{array}{l}\text { Bilateral Middle Ear } \\
\text { Cholesteatoma }\end{array}$ & Genotype of ABCC11 538>A & Frequency \\
\hline Dry & A/A & $3 / 14$ & $21.4 \%$ \\
\hline Wet & G/A & $7 / 17$ & $41.1 \%$ \\
\hline
\end{tabular}

Table 1: One single nucleotide polymorphism (SNP), 538G>A (Gly180Arg), in the ABCC11 gene determines earwax type.

Few populations of South-east Asian (0-30\%), such as Japanese,

Chinese, and Korean, have the wet type of earwax. The incidence of middle ear cholesteatoma was higher in Europeans, who exhibit a higher frequency of wet earwax, than in the Japanese, who exhibit a low frequency of wet earwax $[1,13]$. In our study, the frequency of wet earwax in patients with middle ear cholesteatoma was $54.8 \%(17 / 31$ patients), suggesting the possibility that the wet type of earwax was higher in patients than in healthy subjects $(<30 \%)[5,13,14]$. Because we could not examine the genotypes of $A B C C 11$ in healthy subjects, we compared our data with results from a previous study that conducted in Japan, in which $15.9 \%$ (59/372) of healthy subjects exhibited wet earwax [5]. The integrated analysis showed that the middle ear cholesteatoma group exhibited a significantly higher incidence of wet earwax $(54.8 \%, 17 / 31$ patients) than the healthy group (15.9\%, 59/372 subjects; $p<0.001)$.

With regard to the incidence of cases with bilateral middle ear cholesteatoma (including past history of surgery for middle ear cholesteatoma), our study demonstrated that there were more individuals with wet earwax (41.4\%, $7 / 17$ patients) than in the group with dry earwax $21.4 \%$ (3/14 patients), However, we could not find a statistically significant difference between the groups, probably due to 
Citation: $\quad$ Kusunoki T, Nakagawa H, Tsukamoto M, Toyoda Y, Kamiya K, et al. (2018) Possible Association between Middle Ear Cholesteatoma and A Single Nucleotide Polymorphism 538G>A in ABCC11, A Human Earwax Type Determinant. J Mol Genet Med 12: 362. doi: $10.4172 / 1747-0862.1000362$

Page 3 of 3

the small number of patients (i.e., $\mathrm{n}=31$ ) who underwent $A B C C 11$ genotyping. We previously described the incidence of cases with bilateral middle ear cholesteatoma according to phenotype and reported that incidence in the group with wet earwax was significantly higher than in the group with dry earwax (10). Under normal physiological conditions, the external auditory canal benefits from antimicrobial agents in ear wax $[15,16]$. Excessive pooling of earwax, however, has may cause discomfort, including itchiness or a sense of fullness in the ear, resulting in repetitive cleaning with chronic irritation of the external auditory canal. As a result, excessive pooling of wet earwax could lead to bilateral middle ear cholesteatoma.

Earwax, which contains lipids and proteins, is defined as a mixture of secretions from the apocrine glands in the extra-auditory canal. Since non-functional $A B C C 11$ protein arises from the $A B C C 11$ gene with the 538A contrary to the case with the $538 \mathrm{G}$ allele, genetic-based differences in those human phenotypes of earwax are likely due to the presence of functional $A B C C 11$ proteins [3,4]. According to a previous study addressing the constituents of fatty acids (as a major component of lipids) in wet and dry earwax samples, wet earwax could contain some unknown fatty acids [17]. Due to differences in the components and volume of lipids between dry and wet earwax, the lipids in wet earwax arising from the apocrine glands expressing functional $A B C C 11$ protein might cause greater oxidative damage than that of dry earwax.

\section{Conclusion}

Dara from the present study and other reports suggest that a SNP $A B C C 11538 \mathrm{G}>\mathrm{A}$, a human earwax type determinant, might influence the physiological environment of the external auditory canal and result in an increased incidence of middle ear cholesteatoma. Factors in the external auditory canal responsible for middle ear cholesteatoma could include wet earwax relating to the functional $A B C C 11$. Further studies with a larger number of subjects are needed to determine whether wet earwax is a risk factor for bilateral cholesteatoma.

\section{Declarations}

\section{Ethics approval and consent to participate}

Blood samples were collected from the patients under oral and written informed consent. The present research was approved by the Juntendo University Committee for Ethics related to human genome.

\section{Competing interests}

The authors have no conflict of interest to declare.

\section{Funding}

This study was supported by a Gant-in-Aid for Scientific Research (C) (JSPS KAKENHI Grant Number 15K10763, 18K09356) to T.K. from the Japan Society for the Promotion of Science (JSPS).

\section{Author's contributions}

Takeshi Kusunoki conceived and designed this study. Hiroshi Nakagawa and Yu Toyoda assisted with research design. Kazusaku
Kamiya, Hiroko Okada, Satoshi Hara and Masayuki Furukawa collected samples and analyzed clinical data. Hiroshi Nakagawa, Megumi Tsukamoto and Yu Toyoda involved in the genetic analysis. Takeshi Kusunoki wrote the manuscript. Hiroshi Nakagawa, Yu Toyoda, Kazusaku Kamiya and Katsuhisa Ikeda provided intellectual input and assisted with the preparation of the manuscript.

\section{Acknowledgements}

The authors thank Mr. Brent Bell for his reviewing this manuscript.

\section{References}

1. Nakagawa H, Toyoda $\mathrm{Y}$, Albrecht T, Tsukamoto M, Paraetoirus M, et al. (2018) Are ATP-binding cassette transporter and earwax associated with the incidence of cholesteatoma? Med Hypotheses 114: 19-22.

2. Yoshiura K, Kinoshita A, Ishida T, Ninokata A, Ishikawa T, et al. (2006) A SNP in the $A B C C 11$ gene is the determinant of human earwax type. Nat Genet 38: 324-330.

3. Toyoda Y, Sakurai A, Mitani Y, Nakashima M, Yoshiura K, et al. (2009) Earwax osmidrosis, and breast cancer: why does one SNP $(538 \mathrm{G}>\mathrm{A})$ in the human $\mathrm{ABC}$ transporter $A B C C 11$ gene determine earwax type? FASEB J 23: 2001-2013.

4. Toyoda Y, Takada T, Gomi T, Nakagawa H, Ishikawa T, et al. (2017) Clinical and molecular evidence of $A B C C 11$ protein expression in axillary apocrine glands of patients with axillary osmidrosis. Int J Mol Sci 18: 417.

5. Takagi T, Hato N, Gyo K (2011) Earwax genotype as a factor of middle ear cholesteatoma. Otol Jpn 21: 791-794.

6. Tos M (1988) Incidence, etiology and pathogenesis of cholesteatoma in children. Adv Otorhinolaryngol 40: 110-117.

7. Padgham N, Mills R, Christmas H (1989) Has the increasing use of grommets influenced the frequency of surgery for cholesteatoma? J Laryngol Otol 103: 1034-1035.

8. Kemppainen HO, Puhakka HJ, Laippala PJ, Sipila MM, Manninen MP, et al. (1999) Pidemiology and etiology of middle ear cholesteatoma. Acta Otolaryngol 119: 568-672.

9. Britze A, Moller ML, Ovesen Y (2017) Incidence of a 10-year recidivism rate and prognostic factors for cholesteatoma. J Laryngol Otol 131: 319-328.

10. Kusunoki T, Nakagawa H, Tsukamoto M, Toyoda Y, Kamiya K, et al. (2017) Earwax phenotype related to risk factor of middle ear cholesteatoma. M J Otol 2: 003.

11. Sade J, Luntz M, Levy D (1995) Middle ear gas composition and middle ear aeration. Ann Otol Rhinol Laryngol 104: 369-373.

12. Takahashi H, Honjo I, Naito Y, Miura M, Tanabe M, et al. (1995) Gas exchange function through the mastoid mucosa in ears after surgery. Laryngoscope 107: 1117-1121.

13. Ishikawa T, Toyoda Y, Yoshiura K, Niikawa N (2012) Pharmacogenetics of human $\mathrm{ABC}$ transporter $A B C C 11$ : New insights into apocrine gland growth and metabolite secretion. Front Genet 3: 1-17.

14. Sato T, Ono H, Ishida H, Kodera H, Matsumura H, et al. (2009) Allele frequencies of the $A B C C 11$ gene for earwax phenotypes among ancient populations of Hokkaido, Japan. J Hum Genet 54: 409-413.

15. Chai TJ, Chai TC (1980) Bactericidal activity of cerumen. Antimicrob Agents Chemother 18: 638- 641.

16. Lum CL, Jeyanthi S, Prepageran N, Vadivelu J (2009) Antibacterial and antifungal properties of human cerumen. J Laryngol Otol 123: 375-378.

17. Iwsaki S, Suzuki H, Moteki H, Takumi Y, Usami S (2011) Analysis of fatty acids from wet and dry wax samples. Otologia Fukuoka 257: 35-41. 